\title{
Levofloxacino frente a betalactámicos en el tratamiento de la neumonía adquirida en la comunidad con ingreso hospitalario
}

\author{
Mª P. GEIJO MARTÍNEZ, A. M. DÍAZ DE TUESTA CHOW-QUAN, \\ C. ROSA HERRANZ, C. GÓMEZ CRIADO*, J. F. DIMAS NUÑEZ, \\ F. SAIZ GARCÍA
}

Unidad de Enfermedades Infecciosas. Servicio de Medicina Interna. Sección de Microbiología. *Laboratorio de Análisis Clínicos. Hospital General. Cuenca

\begin{abstract}
LEVOFLOXACIN VERSUS BETALACTAMIC THERAPY IN COMMU NITY-ACQUIRED PNEUMONIA THAT REQUIRES HOSPITALIZA TION
\end{abstract}

\section{RESUMEN}

Objetivo: Comparar la eficacia y seguridad de levofloxacino frente al tratamiento estándar con betalactámicos en pacientes con Neumonía Adquirida en la Comunidad que cumplen criterios de ingreso hospitalario $(\mathrm{NACH})$.

Pacientes y métodos: Estudio prospectivo de un año de duración de 49 pacientes (ptes.) ingresados en el Servicio de Medicina Interna, diagnosticados de NACH con asignación aleatoria a recibir.- I: cefotaxima o ceftriaxona, II: amoxicilina-clavulánico, (las dos previas asociadas o no a un macrólido) o, III: levofloxacino. Se realizó Rx. tórax a los 7 -10 días, al mes y, posteriormente si era necesario.

Resultados: 29 ptes. recibieron pauta estándar (I o II) y 20 ptes. levofloxacino. Eran varones el 84\%, con edad media de 70,9 años, 57\% con enfermedad de base moderada o grave, y $55 \%$ con criterios de gravedad inicial. En el 47 \% de los casos se llegó a diagnóstico etiológico, siendo en un tercio de los casos bacilos gramnegativos. Se produjo la curación en el $94 \%$ de los pacientes y éxitus en 2 pacientes (5\%). No se observaron diferencias entre los dos grupos en las características demográficas, enfermedad de base ni gravedad inicial. No se detectaron diferencias en los efectos secundarios, complicaciones, estancia hospitalaria o porcentaje de curaciones. La necesidad de prolongar el tratamiento fue mayor en el grupo estándar que en el tratado con levofloxacino (52 vs $15 \%$, p: $0,02)$.

Conclusiones: En población mayor y con gravedad inicial el tratamiento con levofloxacino puede ser una alternativa válida al tratamiento estándar.

PALABRAS CLAVE: Neumonía adquirida en la comunidad. Tratamiento antibiótico. Levofloxacino.

\section{ABSTRACT}

Objective: To compare the effectiveness and security of levofloxacin treatment in front betalactamic therapy in patient with community-acqui red pneumonia that require hospitalization $(C A P H)$.

Patient and methods: A prospective and randomized study along a year from 49 patients diagnosed of $(C A P H)$ that were admitted in the Internal Medicine Service. The patients were assigned randomly to receive. I: cefotaxime or ceftriaxone, II: amoxicillin/ clavulanate (both could be associated or not with a macrolide) or III: levofloxacin. It was accomplished Rx. thorax to $7-10$ days, to the month and, other reviews if was necessary.

Results: 29 cases were in standard therapy (I or II) and 20 cases received levofloxacin therapy. Male $84 \%$, half age 70,9 years old, $57 \%$ with moderate or severe underlying disease, and $55 \%$ with approaches of initial severity criteria. In $47 \%$ of the cases we arrived to etiologic diagnosis, in the third of the cases were BGN. The cure took place in 94 $\%$ of the patients and 2 patients died (5\%). No differences were observed regarding demographic characteristics, underlying disease and severity. No differences were detected in: the secondary effects, complications, hospital stay or, mean stay or percentage of cures. The necessity to pro long the therapy was bigger in the standard group in front of the group tried with levofloxacin (52\% vs. $15 \%$, p:0.02).

Conclusions: In bigger population and with initial severity the treat ment with levofloxacin can be a valid alternative to the standard therapy.

KEY WORDS: Community-acquired pneumonia. Antibiotic treatment. Levofloxacin.

Geijo Martínez MP, Díaz de Tuesta Chow-Quan AM, Rosa Herranz C, Gómez Criado C, Dimas Nuñez JF, Saiz García F. Levofloxacino fren te a betalactámicos en el tratamiento de la neumonía adquirida en la comunidad con ingreso hospitalario. An Med Interna (Madrid) 2002; 19: 621-625.

\section{INTRODUCCIÓN}

La necesidad de seleccionar el tratamiento antibiótico empírico de las neumonías adquiridas en la comunidad (NAC) se basa en: datos de la historia, datos epidemiológicos locales, enfermedades concurrentes, gravedad de la infección y los hallazgos de la tinción Gram del esputo. El tratamiento actual de la NAC se realiza con prescripción de forma individual o combinada de dos grupos antibióticos: betalactámicos y macrólidos. Aunque la rápida expansión del número de agentes antimicrobianos se ha acompañado de numerosos estudios sobre su eficacia (1).

Trabajo aceptado: 28 de agosto de 2002

Correspondencia: Ma . Paloma Geijo Martínez. Servicio de Medicina Interna. Hospital General de Cuenca. C/ Hermandad de Donantes de Sangre, 1. 16002 Cuenca. e-mail: medicinainterna.cuenca@ citelan.es 
Ciertas quinolonas como ofloxacino y ciprofloxacino tienen una cierta utilidad en el tratamiento de la NAC por su actividad frente a $H$. influenzae, BGN entéricos, bacterias intracelulares y L. pneumophila. Pero no eran antibióticos de elección para el tratamiento empírico de la NAC dada su reducida actividad frente al neumococo y otros grampositivos. Los nuevos integrantes del grupo (como levofloxacino, moxifloxacino y, especialmente esparfloxacino, clinafloxacino y grepafloxacino) poseen una excelente actividad frente a los cocos grampositivos, incluidas las cepas de neumococo con alta resistencia, lo que a priori les convierte en una alternativa potencial a las pautas actuales (1). Sí parece claro que serian el tratamiento de elección en caso de alergia a betalactámicos, por la posible pérdida de eficacia de los macrólidos en el tratamiento de las infecciones neumocócicas $(2,3)$.

El levofloxacino es muy activo frente a gérmenes gramnegativos y grampositivos y bacterias atípicas e intracelulares, aunque su acción sólo es moderada frente a gérmenes anaerobios (4). Los efectos adversos más comunes son: náuseas, diarrea, alteraciones hepáticas, convulsiones y colitis pseudomembranosa. Administrado por vía intravenosa (i.v.) es eficaz para el tratamiento de pacientes con NAC y administrado por vía oral (v.o.) tiene biodisponibilidad del $100 \%$, por lo que el cambio precoz de la vía i.v. a la v.o. permite hospitalizaciones más breves y menos costosas (2).

Las recomendaciones respecto al tratamiento empírico en pacientes con NAC con criterios de ingreso han cambiado en los últimos años, tanto Centers of Disease Control and Pre vention (CDC) como el comité de la Sociedad Americana de Enfermedades Infecciosas (Infectious Disease Society of Ame rica) (IDSA) consideran las fluorquinolonas en el tratamiento de primera línea en pacientes con criterios de ingreso en la neumonía adquirida en la comunidad 5 porque son activas frente a casi todos los agentes no virales que producen neumonía, principalmente $S$. pneumoniae y Legionella (las dos principales causas de mortalidad de la NAC).

Las nuevas fluorquinolonas son comparables al tratamiento estándar con amoxicilina-clavulánico o cefalosporinas en el tratamiento de pacientes ambulatorios con NAC leve o moderada. Sin embargo, pocos estudios clínicos bien diseñados han sido publicados para evaluar el papel de las primeras en el tratamiento de pacientes con NAC severa que requieren hospitalización o ingreso en la Unidad de Cuidados Intensivos $(6,7)$.

En este estudio prospectivo, aleatorio y controlado se pretende comparar la eficacia y seguridad del tratamiento estándar frente al tratamiento con levofloxacino en pacientes con $\mathrm{NAC}$ con criterios de ingreso hospitalario (NACH).

\section{PACIENTES Y MÉTODOS}

Durante el periodo del 1 de noviembre del 1999 al 1 de noviembre del 2000 se estudiaron de forma prospectiva, pacientes diagnosticados de NAC con criterios de ingreso hospitalario en el Servicio de Medicina Interna (Hospital Virgen de la Luz, Cuenca) desde el Servicio de Urgencias.

Se incluyeron los pacientes diagnosticados de NAC por síntomas y signos compatibles con infección del tracto respiratorio inferior (tos, expectoración, fiebre, dolor torácico pleurítico, disnea o alteración del estado mental) junto con infiltrados pulmonares en la radiografía de tórax, que no existían previamente ni eran debidos a otra causa, sin que hubiera existido un ingreso hospitalario los siete días previos al inicio de los síntomas. Se excluyeron los pacientes en los que la neumonía era de adquisición nosocomial.

Se consideraron criterios de ingreso hospitalario: edad superior a 60-65 años, patologías crónico-debilitantes (enfermedad pulmonar obstructiva crónica-EPOC-, cirrosis hepática, insuficiencia renal crónica, diabetes mellitus, neoplasia, cardiopatía) o situación de inmunosupresión, criterios de gravedad, ausencia de respuesta a un tratamiento ambulatorio aparentemente correcto iniciado 72 horas antes, afectación radiológica de más de un lóbulo, presencia de cavitación o derrame pleural, sospecha de broncoaspiración o sospecha que el paciente no va a realizar el tratamiento ambulatorio de forma correcta (sociopatía).

Se consideraron criterios de gravedad: inestabilidad hemodinámica (presión arterial sistólica menor $(<)$ de $90 \mathrm{mmHg}$, pulso mayor (>) 125), o $\mathrm{pH}<7,35$; insuficiencia respiratoria $\left(\mathrm{pO}_{2}<60 \mathrm{mmHg}\right.$, cociente $\mathrm{PO}_{2} / \mathrm{FiO}_{2}<$ a $250 \mathrm{mmHg}$, o frecuencia respiratoria $>30$ por minuto); insuficiencia renal aguda; alteración del nivel de conciencia; bacteriemia y/o complicaciones sépticas; temperatura $>40^{\circ} \mathrm{C}$ o $<35^{\circ} \mathrm{C}$; afección radiológica de más de un lóbulo, derrame o cavitación; anemia o leucopenia; y urea $>60$, sodio sérico $<130$, glucemia $>$ 250 o albúmina sérica $<3,1$.

Se excluyeron aquellos pacientes que habían recibido tratamiento antibiótico previo durante más de 48 horas, alérgicos a quinolonas, embarazo, lactancia o tratamiento con anticonvulsivantes; y aquellos con criterios de gravedad, que requerían ingreso en la Unidad de Cuidados Intensivos: alteración importante de la consciencia, insuficiencia respiratoria grave (frecuencia respiratoria $>35$ por minuto, utilización de la musculatura accesoria, fatiga muscular diafragmática, cociente $\mathrm{PO}_{2} / \mathrm{FiO}_{2}<250 \mathrm{mmHg}$ o $<200 \mathrm{mmHg}$ en EPOC), inestabilidad hemodinámica grave (requerimiento de drogas vasoactivas durante más de cuatro horas, diuresis horaria $<20 \mathrm{ml} / \mathrm{h}$ o en 4 horas $<80 \mathrm{ml}$ en ausencia de hipovolemia renal), coagulación intravascular diseminada, insuficiencia renal aguda o meningitis.

En el protocolo de recogida de datos se incluyeron las siguientes variables: edad, sexo, procedencia, antecedentes, indicación de ingreso, criterios de gravedad, tipo de tratamiento, síntomas, exploración física, pruebas de laboratorio, radiografía, complicaciones, microbiología y evolución. En todos los pacientes -al ingreso y previo a la instauración del tratamiento antibiótico-, se les realizó: hemograma, bioquímica, sedimento de orina, gasometría arterial (según indicación del clínico), hemocultivos (2) y examen bacteriológico de esputo (gram y cultivo). En caso de sospecha de tuberculosis (TB), Ziehl en esputo y si cumplía criterios de neumonía atípica, antígeno de Legionella en orina. En las primeras 48 horas, se realizaron serologías para $M$ pneumoniae, Chlamydia sp, Coxiella burnetti y Legionella sp. Consideramos serología positiva la elevación de cuatro veces el título de la segunda muestra respecto de la primera (diagnóstico por seroconversión), o bien un título único de Ig G mayor o igual a 1/256 para Mycoplasma pneumoniae, Ig G mayor o igual a 1/512 para Coxiella burnetii, Legionella pneumophila, Chlamydia spp. Para el diagnóstico etiológico de $\mathrm{S}$. pneumoniae y el resto de bacterias, consideramos positivo sólo el esputo de calidad con más de 15 pmn y escaso número de células epiteliales y se realizaron métodos invasivos (catéter telescopado, BAL o punción transtorácica) en pacientes con neumonía grave y 
riesgo de padecer etiología no habitual o con falta de respuesta al tratamiento empírico.

Se establecieron tres pautas de tratamiento que se asignaron aleatoriamente a los pacientes, en base a una tabla de números aleatorios establecida en el servicio de urgencias: Pauta I: cefalosporina de $3^{\mathrm{a}}$ generación (cefotaxima 1-2 gr cada 6-8 horas iv o ceftriaxona 1-3 gr al día iv). Pauta II: amoxicilina-clavulánico 2-0,2 gr cada 8 horas, iv. Pauta I y II asociados a un macrólido (azitromicina $500 \mathrm{mg}$ vo 24 horas o claritromicina $250-500 \mathrm{mg}$ vo cada 12 horas) o iv si se sospecha de neumonía atípica. pauta III: levofloxacino $500 \mathrm{mg}$ cada 24 horas iv en monoterápia, pudiendo pasar de forma secuencial a terapia oral.

El seguimiento se realizó tanto clínico como analítico los días 1, 3, y 7 ó10; con radiografía de tórax al ingreso y a los 7 -10 días. Se revisaron los pacientes ambulatoriamente el $28^{\circ}$ día con analítica elemental, segunda muestra serológica y nueva radiografía de tórax y, posteriormente revisiones mensuales si no se producía la curación. Los criterios de evaluación de la respuesta clínica y radiológica fueron: a) curación (desaparición total de la clínica así como del infiltrado radiológico); b) mejoría (la clínica mejoró pero no desaparecieron los signos radiológicos de neumonía); c) fallo terapeútico (persisten o se agravan los signos clínicos y/o radiológicos -derrame, cavitación o algún otro signo de progresión-, y es necesario realizar un tratamiento alternativo).

Análisis estadístico: Se empleó el programa el programa SPSS ${ }^{\circledR}$ versión 8.0. En el análisis descriptivo se emplearon las diferencias simples. Para establecer las diferencias entre las variables cualitativas se utilizó la prueba X2, empleando corrección de Yates. Se utilizó el test de ANOVA para establecer la diferencia entre variables cuantitativas (diferencia entre medias). Se consideró significativo un valor de $\mathrm{p}<0,05$.

\section{RESULTADOS}

Se trataron un total de cuarenta y nueve pacientes diagnosticados de NAC con asignación aleatoria para recibir tratamiento: 29 pacientes con tratamiento estándar (cefalosporinas de tercera generación o amoxicilina-clavulánico, asociados o no a un macrólido); y 20 pacientes tratados con levofloxacino en monoterápia. El $84 \%$ fueron varones, con una edad media de 70,9 años. El $57 \%$ presentaba una enfermedad de base moderada o grave y el $59 \%$ criterios de gravedad inicial. No había diferencia significativa en las características basales: Edad, sexo, enfermedad de base o gravedad inicial, entre los dos grupos de tratamiento (Tabla I); tampoco era significativa la diferencia entre los distintos criterios de ingreso: Senilidad, enfermedad crónica ó grave, inmunosupresión o criterios de gravedad. Analizando los distintos criterios de gravedad que tenían los pacientes en el momento del diagnóstico de neumonía: Inestabilidad hemodinámica, insuficiencia respiratoria, alteración del nivel de conciencia, bacteriemia o complicaciones sépticas, afección radiológica complicada o alteraciones analíticas, tampoco era significativa la diferencia (Tabla II).

En 23 pacientes $(47 \%)$ se llegó al diagnóstico etiológico: Chlamydia $p$. y BGN fueron los agentes más frecuentes, con un $22 \%$ cada uno, Pseudomona $a$. el $17 \%$, neumococo en un $13 \%$ y Legionella y Coxiella Burnetii en un $9 \%$ respectivamente (Tabla III).

Se produjo la curación en 46 pacientes $(94 \%)$, en 3 pacientes $(6 \%)$ ocurrió un fallo terapéutico, en 2 pacientes se trató de
TABLA I

CARACTERÍSTICAS BASALES DE LOS PACIENTES CON NACH TRATADOS CON LEVO FLO XACINO FRENTE AL GRUPO ESTÁNDAR

\begin{tabular}{lccc}
\hline & Levo & Estándar & $\mathrm{p}$ \\
\hline Edad (años) & 71,8 & 70 & 0,9 \\
Sexo (mujeres) & $20 \%$ & $14 \%$ & 0,7 \\
Enfermedad de base & & & \\
(moderada / grave) & $65 \%$ & $52 \%$ & 0,5 \\
Gravedad inicial & $55 \%$ & $59 \%$ & 0,8 \\
\hline
\end{tabular}

\section{TABLA II}

CAUSAS DE INGRESO HOSPITALARIO DE LOS PACIENTES CON NACH Y CRITERIOS DE GRAVEDAD. ESTUDIO COM PARATIVO ENTRE LAS 2 PAUTAS

\begin{tabular}{lrrc}
\hline Causa de ingreso & Levo & Estándar & $\mathrm{p}$ \\
\hline Senilidad & $30 \%$ & $52 \%$ & 0,2 \\
Enfermedad crónica o grave & $80 \%$ & $62 \%$ & 0,2 \\
Inmunosupresión & $10 \%$ & $7 \%$ & 0,3 \\
Criterios de gravedad & $55 \%$ & $69 \%$ & 0,5 \\
& & & \\
Criterios de gravedad: & & & \\
Inestabilidad hemodinámica & $10 \%$ & $7 \%$ & 0,9 \\
Insuficiencia respiratoria & $35 \%$ & $52 \%$ & 0,4 \\
Alteración del nivel de conciencia & $20 \%$ & $10 \%$ & 0,6 \\
Bacteriemia / complicaciones sépticas & $5 \%$ & $10 \%$ & 0,9 \\
Afección radiológica complicada & $15 \%$ & $7 \%$ & 0,7 \\
Alteraciones analíticas & $15 \%$ & $14 \%$ & 0,8 \\
\hline
\end{tabular}

TABLA III

FRECUENCIA DE PATÓ GENOS AISLADOS

\begin{tabular}{lcc}
\hline Agente etiológico & \multicolumn{2}{c}{$\begin{array}{c}\text { Diagnóstico } \\
\text { etiológico }\end{array}$} \\
& $\mathrm{N}$ o & $(\%)$ \\
\hline S. pneumoniae & 3 & 13 \\
BGN & 5 & 22 \\
Pseudomona sp. & 4 & 17 \\
Staphylococcus & 1 & 4 \\
Streptococcus / Enterococcus & 1 & 4 \\
Chlamydia & 5 & 22 \\
Legionella & 2 & 9 \\
Fiebre Q & 2 & 9
\end{tabular}

BGN (bacilos gramnegativos), № (número),\% (porcentaje).

fallo precoz y el tercero un fallo tardío. Dos pacientes $(5 \%)$ presentaron éxitus, en un caso se trato de mortalidad precoz ( $5^{\circ}$ día de tratamiento) y en el otro caso mortalidad tardía (al $30^{\circ}$ día) en un reingreso con broncoespasmo severo con curación del anterior proceso de neumonía. La estancia media fue de 12,4 días (en el grupo de levofloxacino 10,9 días y en el grupo de tratamiento estándar de 13,8 días (diferencia no significativa). La media de seguimiento de 29,7 días (Tabla IV). 
Las complicaciones extrapulmonares fueron: colescistitis aguda alitiásica ( 1 caso en el grupo estándar), arritmia cardíaca con ICC ( 2 ptes. en el grupo estándar y 1 pte. en el de levofloxacino) y, sepsis con CID (1 pte. en el grupo estándar y otro en el grupo de levofloxacino). Los efectos secundarios fueron: Diarrea por cocos Gram-positivos en 1 paciente en el grupo de levofloxacino y 2 pacientes en el grupo estándar (uno presentó exantema el $10^{\circ}$ día de tratamiento y otro aumento de la bilirrubina sérica en analítica del $7^{\circ}$ día).

No se apreciaron diferencias significativas entre el levofloxacino y el tratamiento estándar en cuanto a: complicaciones extrapulmonares, efectos secundarios, estancia hospitalaria o porcentaje de curaciones. Se prolongó el tratamiento más de diez días de forma significativa en el grupo estándar frente al grupo tratado con levofloxacino: 52 vs $15 \%$, p: 0,02 (Tabla IV). Ninguno de los pacientes con diagnóstico bacteriológico del grupo de levofloxacino presento fallo terapéutico vs un $8 \%$ en el grupo de tratamiento estándar (Fig. 1).

\section{TABLA IV}

EVOLUCIÓN DE LOS PACIENTES CON NACH. COM PARACIÓN DE LAS DOS PAUTAS

\begin{tabular}{lccc}
\hline & Levo & Estándar & $\mathrm{p}$ \\
\hline Complicaciones extrapulmonares & $10 \%$ & $15 \%$ & 0,8 \\
Efectos secundarios & $5 \%$ & $7 \%$ & 0,7 \\
Estancia hospitalaria (días) & $10,9 \%$ & $13,8 \%$ & 0,7 \\
Prolongación tto. (>10 días) & $15 \%$ & $52 \%$ & 0,02 \\
Fallo terapéutico & $5 \%$ & $10 \%$ & 0,6 \\
Exitus & $5 \%$ & $3 \%$ & 0,7 \\
\hline
\end{tabular}

tto.: tratamiento. Levo: levofloxacino.

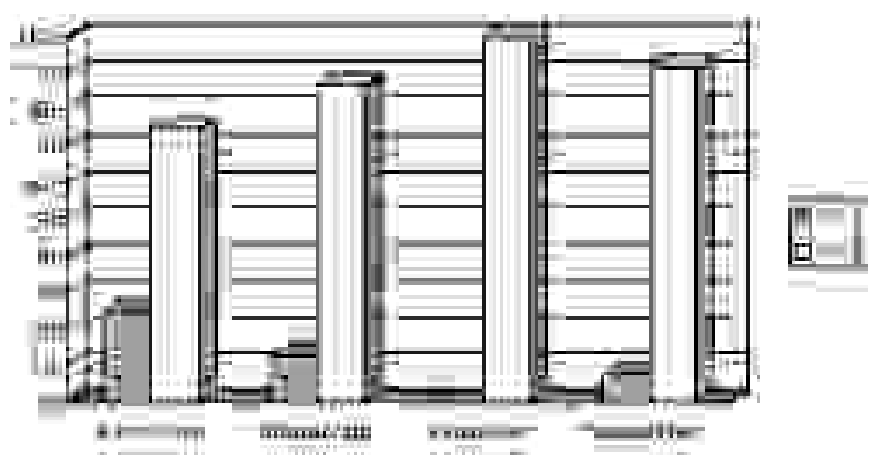

Fig. 1. Fallo terapéutico. Comparación de pacientes con diagnóstico clínico y bacteriológico (Levofloxacino vs estándar).

\section{DISCUSIÓN}

El tratamiento de la NACH actualmente está basado en una terapia empírica; siendo importante la rápida administración de un tratamiento antibiótico para la buena evolución del proceso. Nos apoyaremos en factores epidemiológicos y características microbiológicas locales (8-10). El estudio bacteriológico del esputo, los hemocultivos o la serología tienen baja rentabilidad, en nuestro estudio introdujimos la técnica del antígeno de Legio nella en orina, a pesar de lo cual no encontramos gran porcentaje de este germen a pesar de nuestra vecindad a Levante.
La aproximación inicial para el manejo de los pacientes con NACH incluye valorar el lugar de instauración del tratamiento, la presencia de enfermedad cardiopulmonar y la severidad de presentación de la enfermedad (leve, moderada o severa) (11). Respecto al porcentaje de éxitus en nuestros pacientes $(5 \%)$, dependería más de factores como la gravedad de la enfermedad de base o la senilidad (debemos considerar que nuestro servicio no tiene hospitalización de geriatría, por lo que este tipo de pacientes está incluido en nuestra serie de medicina interna), a pesar de lo cual se trata de una baja mortalidad comparada con otras series (12). Además, dentro del grupo de pacientes con diagnóstico bacteriológico positivo (diagnóstico de neumonía más seguro), en los tratados con levofloxacino no hubo ningún caso de fallo terapeútico.

También tiene importancia la presencia de factores de riesgo para presentar neumococos resistentes a fármacos, o bacterias gramnegativas entéricas (incluyendo P. aeruginosa) (13), porque el tratamiento inicial antibiótico deberá cubrir la mayoría de los patógenos probables para cada caso (14-16). Son susceptibles al levofloxacino: S. aureus sensible a meticilina, S. pneumoniae y $S$. pyogenes, la mayor parte de los enterococos, Chlamydia pn. y Mycoplasma pn.; Pseudomonas a. sólo es moderadamente sensible in vitro (17), es difícil saber el papel que jugó este germen en los casos de neumonía curados en nuestra serie. Muchos ensayos clínicos han demostrado la eficacia de las fluorquinolonas en el tratamiento de la NAC leve a moderada $(2,3,5,18)$. En la neumonía grave con presencia de factores de riesgo para presentar etiología no habitual, en el espectro etiológico, además del neumococo (que sigue siendo el agente etiológico más probable), hay que contemplar L. pneumophila, H. influenzae, bacterias de la flora orofaríngea $\mathrm{y}$, en menor medida, algunas enterobacterias y $\mathrm{S}$. aureus. Tampoco es descartable la posibilidad de alguna bacteria atípica como C. pneumoniae o Coxiella burnetii (11), gérmenes que también aparecieron en nuestro espectro etiológico, como correspondía a una serie con porcentaje alto de neumonías graves.

El comité IDSA americano considera las fluorquinolonas en el tratamiento de primera línea en pacientes con criterios de ingreso en la neumonía adquirida en la comunidad, pero hay que tener en cuenta que su flora bacteriana es menos resistente que la de los países de la Europa meridional 17. En la experiencia canadiense existe un aumento de resistencias del $S$. pneumoniae a las fluorquinolonas en la población general con su mayor uso (19), pero sería más un problema que surgiría en asociación con cepas resistentes a múltiples antibióticos $(20,21)$. En España el agente principal de NAC es el S. Pneumoniae $(15,16)$, aunque varía dependiendo de los medios utilizados para el diagnóstico; en un trabajo reciente en el que se utilizaba el antígeno neumocócico en orina aumentó el número de diagnósticos en un 33\% (22), por lo que no sabemos en nuestra serie cuantos casos más podrían estar causados por este germen. La resistencia a penicilina del neumococo en algunas series en nuestro país llega hasta el $50 \%$, si bien las cepas de alta resistencia son aún minoritarias (1), por lo que la mayoría de los pacientes con infecciones por S. pneumoniae todavía pueden tratarse con un betalactámico. Las fluorquinolonas más modernas deben utilizarse prudentemente, y usarse en aquellas indicaciones que ofrezcan una clara ventaja terapéutica (23).

Nuestros resultados sugieren que el levofloxacino es tan eficaz como los betalactámicos asociados o no a macrólidos 
para el tratamiento de pacientes portadores de $\mathrm{NACH}$, los efectos secundarios no fueron importantes, con solo un caso de posible colitis por antibióticos y en general se prolonga menos la duración del tratamiento, probablemente por la posibilidad de completar el tratamiento por vía oral; lo que ofrece mayor comodidad para el paciente, menor riesgo de infecciones por catéter, así como menores cuidados de enfermería y ahorro de costes hospitalarios (24). En las NAC con criterios de ingreso hospitalario y con criterios de gravedad el levofloxacino puede ser una alternativa válida y eficaz al tratamiento estándar. Es probable que a medida que se disponga de más estudios comparativos intrahospitalarios, estos antibióticos usados en monoterapia vayan sustituyendo a las combinaciones actualmente utilizadas.

\section{AGRADECIMIENTOS}

Los autores agradecen al Servicio de Urgencias del Hospital Virgen de la Luz el apoyo prestado en la asignación de los tratamientos y a los residentes de Medicina de familia Lorena Beatobe y Alberto Herranz por su trabajo en la cumplimentación de protocolos.

\section{Bibliografía}

1. Dorca J. Fernández S. Tratamiento de la neumonía comunitaria. Arch Bronconeumol 1998: 34 (Supl. 2): 50-56.

2. Kalbermatter V, Bagilet D, Diab M, Javkin E. Levofloxacino oral versus ceftriaxona y amoxicilina/ ácido clavulánico en el tratamiento de la neumonía adquirida en la comunidad con criterio de ingreso. Med Clin (Barc) 2000; 4; 115 (15): 561-3.

3. Petitpretz P, Arvis P, Marel M, Moita J, Urueta J; CAP5 Moxifloxacin Study Group. Oral moxifloxacin vs high-dosage amoxicillin in the treatment of mild-to-moderate, community-acquired, suspected pneumococcal pneumonia in adults. Chest 2001; 119(1): 185-95.

4. Mandell LA, Marrie TJ, Grossman RF, Chow AW, Hyland RH and the Canadian Community-Adquired Pneumonia Working Group. Canadian guidelines for the initial management of community-acquired pneumonia: An evidence-based update by the Canadian Infectious Disease Society and the Canadian Thoracic Society. Clin Infect Dis 2000; 31: 383-421.

5. Weinstein RA. Practice guidelines make perfect, Part II: Communityacquired pneumonia in adults. 38th Annual Meeting of the Infectious Diseases Society of America. Infectious Disease Conference Summaries 2000. Medscape.

6. Bayas JM, Vilella A, San-Martín M, González A, Conesa A, Asenjo MA. Impacto sanitario de los ingresos por neumonía en un hospital del tercer nivel. Med Clin (Barc) 2001; 116: 694-5.

7. Álvarez Sanchez B, Álvarez-Lerma F, Jordá R, Serra J, López-Cambra MJ, Sandar MD, y Grupo de estudio de neumonía comunitaria grave en España. Factores pronósticos y etiología de los pacientes con neumonía grave ingresados en UCI. Med Clin (Barc)1998; 111: 650-54.

8. Niederman MS, Mandell LA, Anzueto A, Bass JB, Broughton WA, Campbell GD, Dean N, File T, Fine M J, Gross PA, Martinez F, Marrie TJ, Plouffe JF, Ramirez J, Sarosi GA, Torres A, Wilson R, Yu VL. Guidelines for the management of adults with community-acquired pneumonia. Diagnosis, assessment of severity, antimicrobial therapy, and prevention. Am J Respir Crit Care Med 2001; 163: 1730-54.

9. Theerthakarai R, El-Halees W, Ismail M, Solis R A, Khan MA. Nonvalue of the initial microbiological studies in the management of nonsevere community-acquired pneumonia. Chest 2001; 119: 181-4.

10. Fine MJ, Auble TE, Yealy DM, Hanusa BH, Weissfeld LA, Singer DE, Coley CM, Marrie TJ, Kapoor WN. A prediction rule to identify lowrisk patients with Community-acquired Pneumonia. N Engl J Med 1997; 336: 243-50.

11. Fine MJ, Smith MA, Carson CA, Mutha SS, Sankey SS, Weissfeld LA et al Prognosis and outcomes of patients with community-acquired pneumonia. A meta-analysis. JAMA 1996; 275: 134-141.

12. Medrano González F, Solis García del Pozo J, Gomariz García S y Solera Santos J. Neumonia adquirida en la comunidad que requiere ingreso hospitalario. Una comparación del manejo clínico, consumo de recursos y pronóstico entre diferentes especialidades médicas. Rev Clin Esp 2001; 201: 65-68.
13. Grupo de Estudio de la Neumonia Adquirida en la Comunidad Tratada Ambulatoriamente (NACTA). Diagnóstico y tratamiento de pacientes con neumonía adquirida en la comunidad: variabilidad de las pruebas complementarias y estudio de la efectividad de roxitromicina. Rev Clin Esp 1999; 199(11): 700-704.

14. Niederman MS, Mandell LA, Anzueto A, Bass JB, Broughton WA, Campbell GD, Dean N, File T, Fine MJ, et al. Guideliness for the management of adults with Community-adquired pneumonia. American Thoracic Society. Am J Respir Crit Care Med 2001; 163: 1730-45.

15. Álvarez-Sala JL, Serrano R. Neumonía en el anciano. Med Clin (Barc) 2001; 117: 454-456.

16. Álvarez Gutiérrez FJ, García Fernández A, Elías Hernández T, Romero Contreras J, Romero Romero B, Castillo Gómez J. Neumonías adquiridas en la comunidad en pacientes mayores de 60 años. Incidencia de gérmenes atípicos y evolución clinicorradiológica. Med Clín (Barc) 2001; 117: 441-445.

17. Bartlett JG, Dowell SF, Mandell LA, File TM, Jr, Musher DM, Fine MJ. Practice guidelines for the management of community- adquired pneumonia in adults. Clin Infect Dis 2000; 31: 347-82.

18. Hoeffken G, Meyer HP, Winter J, Verhoef L; CAP1 Study Group. The eficacy and safety of two oral moxifloxacin regimens compared to oral clarithromycin in the treatment of community-acquired pneumonia. Respir Med 2001; 95(7): 553-64.

19. Chen DK, McGeer A, de Azavedo JC, Low DE. Decreased susceptibility of Streptococcus pneumoniae to fluoroquinolones in Canada. N Engl J Med 1999; 341: 233-39.

20. Whitney CG, Farley MM, Hadler J, Harrison LH, Lexau C, Reingold A, Lefkowitz L, Cieslak PR, Cetron M, Zell ER, Jorgensen JH, Schuchat A. Increasing prevalence of multidrug-resistant Streptococcus pneumoniae in the United States. N Engl J Med 2000; 343: 1917-1924.

21. Daily P, Gelling L, Rothrock G, Reingold A, Vugia D, Barrett NL, Hadler J, Baughman W, Farley M, Blake PA, Pas MA, Roche J. et al. Resistance of Streptococcus Pneumoniae to Fluoroquinolones --United States, 1995-1999. Centers for Disease Control 2001.MMWR 50(37): 800-804.

22. Mirete Ferrer C, Gutierrez Rodero F, Rodríguez Díaz JC, Royo García G, Shum Fhunk C y Martín Hidalgo A. Etiología de la neumonia adquirida en la comunidad tratada ambulatoriamente. Utilidad de un protocolo diagnóstico con pruebas microbiológicas convencionales y detección de antígenos de Streptococcus pneumoniae y Legionella pneumophila en orina. Med Clín (Barc) 2001; 117: 657-659.

23. Liñares J, de la Campa AG, Pallares R. Fluoroquinolone resistance in Streptococcus pneumoniae. N Engl J Med 1999; 341: 1546-48.

24. Rittenhouse BE, Stinnett AA, Dulisse B, Henke CJ, Potter L, Parasuraman B, Martens LL, Williams RR, Kojak C. An economic evaluation of levofloxacin versus cefuroxime axetil in the outpatient treatment of adults with community-acquired pneumonia. Am J Manag Care 2000; 6(3): 381-9 